\title{
Restoration of Neonatal Retinal Images
}

\author{
Sharath M Shankaranarayana ${ }^{1}$, Keerthi Ram ${ }^{2}$, Anand Vinekar ${ }^{3}$, Kaushik \\ Mitra $^{1}$, and Mohanasankar Sivaprakasam ${ }^{1,2}$ \\ 1 Indian Institute of Technology Madras (IITM), India, \\ 2 Healthcare Technology Innovation Centre, IITM, India, \\ 3 Narayana Nethralaya
}

\begin{abstract}
Retinopathy of prematurity (ROP) is an eye disorder primarily affecting premature neonates. Specialists use a number of neonatal retinal images acquired by a wide field of view camera for diagnosis and the subsequent follow up. However, the premature infants' retinal images are generally of lower visibility compared to adult retinal images, affecting the quality of diagnosis. We study some image dehazing methods from general outdoor scenes and propose an image restoration scheme for neonatal retinal images, based on the physical model of light propagation in a medium. The results from our restoration algorithm is useful for analysis by human experts as well as computer aided diagnosis and specifically we show that our method enhances vessel segmentation significantly compared to traditional methods like adaptive histogram equalization.
\end{abstract}

Keywords: image restoration, retinopathy of prematurity, dehazing, vessel segmentation

\section{Introduction}

Worldwide, increase in neonatal survival rates has led to an increase in risk of Retinopathy of prematurity (ROP), manifested from abnormal vascular development in the eyes of premature babies. ROP could become aggressive with neovascularization and potentially progress to retinal detachment and blindness. Guidelines recommend weekly screening of at-risk neonates using a wide field of view camera to capture and examine retinal images [1] and to detect and stage the progression of the disease, and plan interventions. Challenges in imaging of infant retinas result in lower image quality and information content (Fig. 1) as compared to retinal imaging in adults. Limited dilation, heavy fundus pigmentation, corneal and vitreous haze are some of the reasons attributed to the poor image quality which can be addressed using image restoration techniques.

Single image based dehazing has been proposed for restoring outdoor hazy images [4-6] and underwater images $[7,8]$, however there has not been much in reported literature that address restoration of neonatal retinal images.

The model commonly used for dehazing is based on the Koschmieder model [3] and is given by:

$$
I(\mathbf{x})^{c}=t(\mathbf{x}) J(\mathbf{x})^{c}+(1-t(\mathbf{x})) A^{c}
$$

X. Chen, M. K. Garvin, J. Liu, E. Trucco, Y. Xu (Eds.): OMIA 2016, Held in Conjunction with MICCAI 2016, Athens, Greece, Iowa Research Online, pp. 49-56, 2016. Available from: http://ir.uiowa.edu/omia/2016_Proceedings/2016/ 


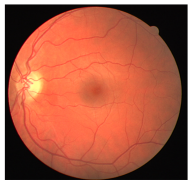

(a). Adult Retinal Image

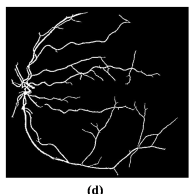

(d)

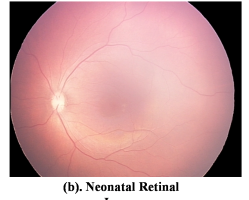

(b). Neonatal Reting
Image

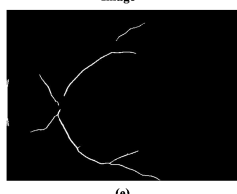

(e)

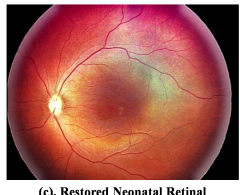

\begin{tabular}{l} 
(c). Restored Neonata \\
Image \\
\hline
\end{tabular}

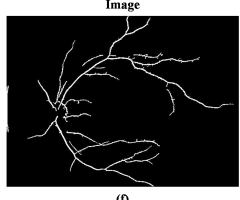

Fig. 1: Figure showing the difference between (a) adult and (b) infant retina and (c) output of our restoration method on (b) and (d), (e), (f) are the outputs of vessel segmentation method of [2], from which it can be clearly seen that our method results in improved segmentation of neonatal retinal images.

where $\mathbf{x}$ is a pixel coordinate, $c$ represents RGB color channel, $I(\mathbf{x})$ is the observed hazy image, $J(\mathbf{x})$ is the original scene radiance, $A$ is the airlight vector, also referred to as ambient light, and $t(\mathbf{x})$ is the transmission map which follows the Beer-Lambert's law, and is given by:

$$
t(\mathbf{x})=\exp (-\mu d(\mathbf{x}))
$$

where $\mu$ is the scattering coefficient of the medium and $d(\mathbf{x})$ represents the depth. The parameters that need to be estimated are $A$ and $t$, given the single observed image $I$, for computing $J$ (dehazed image). To solve for $J$ from single equation, additional priors need to be imposed. One of the solutions [5] is the use of a darkchannel prior to obtain an initial estimate of transmission $t(\mathbf{x})$ and later refine it using soft matting technique. A somewhat similar but faster method for dehazing is proposed in [6] where 'veil' $((1-t(\mathbf{x})) A)$ is inferred instead of transmission, and refined using median of median filtering before inverting equation (1) to obtain $J(\mathbf{x})$.

The above model has been applied only for either natural daytime scenes or underwater images. Such models have not yet been studied for restoration of images in medical domain, specifically retinal imaging. Therefore, we wish to study the effectiveness of the model in neonatal retinal images. The main contributions of this work are-

1. proposing an optical model for ROP imaging similar to the cases of underwater imaging and imaging through fog or haze,

2. providing quantitative insights on viewing the neonatal retinal imaging as imaging through turbid media by comparing with adult retinal imaging.

3. proposing an image restoration technique based on modified version of dark channel prior and experimentally showing the improvement in segmentation after restoration.

\section{Method}

The model described in the previous section is applicable for natural scenes in the presence of atmospheric scattering like haze or fog. Such images generally 


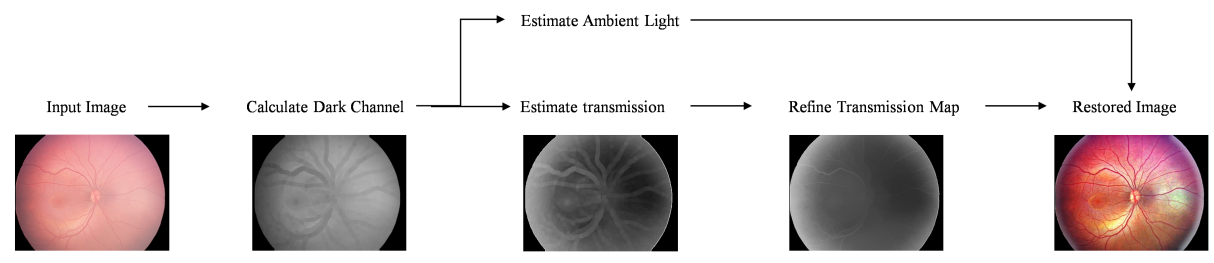

Fig. 2: Flowchart of the method

have far objects, nearly planar objects, ambient light source illuminating in arbitrary orientation with respect to camera pose. Some of the observations that differentiate the case of retinal imaging from the above are i)since the medium in the eye is mostly composed of vitreous humor, the scattering effects can be assumed to be uniform throughout the eye and ii) since the camera uses artificial illumination, the interaction of light among the scatterers causes some amount of scattered light from foreground and background to be re-scattered towards the camera, similar to airlight or ambient light. With the aforementioned points in mind, the captured image is written as:

$$
I(\mathbf{x})^{c}=I(\mathbf{x})^{c}{ }_{D}+I(\mathbf{x})^{c}{ }_{B S}
$$

where $I(\mathbf{x})^{c}{ }_{D}$ represent the direct component and $I(\mathbf{x})^{c}{ }_{B S}$ represents the backscattering component. The direct component can be further decomposed as:

$$
I(\mathbf{x})^{c}{ }_{D}=t(\mathbf{x}) L^{c} J(\mathbf{x})^{c}
$$

where $J(\mathbf{x})$ represents the reflectance and $L$ represents the luminance and $\mathrm{t}(\mathbf{x})$ represents the transmission map. Also the backscattering component can be decomposed as:

$$
I(\mathbf{x})^{c}{ }_{B S}=(1-t(\mathbf{x})) A^{c}
$$

where $A^{c}$ represents the ambient light. Thus, it can be seen that the original scene is degraded by two components- the multiplicative component i.e transmission map which causes attenuation and the additive component i.e the backscattering component which produces a veiling effect resulting in further degradation. In this case, it can be noted that there is only one light source and all the regions in the retina are illuminated by the same light source. Moreover, considering low variation in the depth and the fact that there is only one source of illumination, we can consider that the line of sight and the retinal surface are all illuminated by the same source and thus ambient light in the backscattering component is same as the source for illumination and have $L=A$, and therefore the final equation for the modified model becomes:

$$
I(\mathbf{x})^{c}=t(\mathbf{x}) A^{c} J(\mathbf{x})^{c}+(1-t(\mathbf{x})) A^{c}
$$

The equation (6) has unknowns in $t(\mathbf{x}), A$ and $J(\mathbf{x})$. For getting the dehazed image, we need to have good estimates of $t(\mathbf{x})$ and $A$. Based on the observation that haze-free outdoor images have very low intensities in a square patch in at least one color channel, He et. al [5] introduce a novel prior called dark channel 
prior, given mathematically by

$$
J^{\text {dark }}(\mathbf{x})=\min _{c \in(r, g, b)}\left(\min _{\Omega(x)}\left(J(\mathbf{x})^{c}\right)\right.
$$

where $J^{\text {dark }}$ is the dark channel of the image $J$ and $\Omega(x)$ is a local patch centered around $x$. The authors collect a large number of images from the Internet and from the histogram of dark channel of the images, they show that about 75 percent of the pixels have zero values, and the intensity of 90 percent of the pixels is below 25. They make an observation that for an outdoor haze free image, intensity of $J$ is low and $J^{\text {dark }}$ tends to zero, except for the sky regions. A hazy image is brighter than its haze-free version due to additive airlight. Assuming $A$ is given, the authors use the concept of dark channel prior to derive the initial estimate of $\tilde{t}(\mathbf{x})$. Formally, the initial transmission estimate is given by:

$$
\tilde{t(\mathbf{x})}=1-\min _{c \in(r, g, b)}\left(\min _{\Omega(x)}\left(\frac{I(\mathbf{x})^{c}}{A^{c}}\right)\right)
$$

This coarse estimate is later refined using spectral matting techniques. The airlight $A$ is also estimated from the dark channel by first picking the top 0.1 percent of brightest pixels in the dark channel and among these pixels selecting the pixel with highest intensity in $I$ as the airlight, the idea being that the brightest pixels in the dark channel are affected most by airlight.

We propose a similar approach to get the transmission estimate but with some important modifications. In outdoor dehazing models as well as our model, we only consider the effects of scattering. It is known that scattering and absorption are different phenomena. The former depends only on the scene depth whereas the latter is a function of both scene depth and wavelength. In the case of retinal imaging, the green light is mostly absorbed by the vessels and only partially reflected. Therefore, we consider the green channel to be absorptive and hence in our model we consider only the red and blue channels for calculating the dark channel.

$$
J^{\text {dark }}(\mathbf{x})=\min _{c \epsilon(r, b)}\left(\min _{\Omega(x)}\left(J(\mathbf{x})^{c}\right)\right.
$$

Before proceeding further we would like to test the validity of this dark channel prior in retinal imaging. For this, we first compute the dark channels of adult retinal images from STARE database and neonatal retinal images from a local hospital. We plot the histogram of average intensities of dark channels of all the images, histogram of intensities of all the pixels in dark channel and corresponding CDF of all 397 images from STARE (top row of Fig. 2) and 325 images from commercially available hand-held neonatal imaging device (bottom row of Fig. 2). The histograms indicate that dark channels of infant retinal images are overall brighter ( $80 \%$ of pixels have intensity values higher than 50 ) than those of adult retinal images (showing $90 \%$ of pixels have intensities less than 50). This shift in the intensities of neonatal retinal images is attributed to 'ambient light' (due to turbid medium) and therefore validates the use of dark channel prior in neonatal retinal imaging, with the dark channel of adult retinal images as reference. This observation further strengthens our initial claim of viewing 

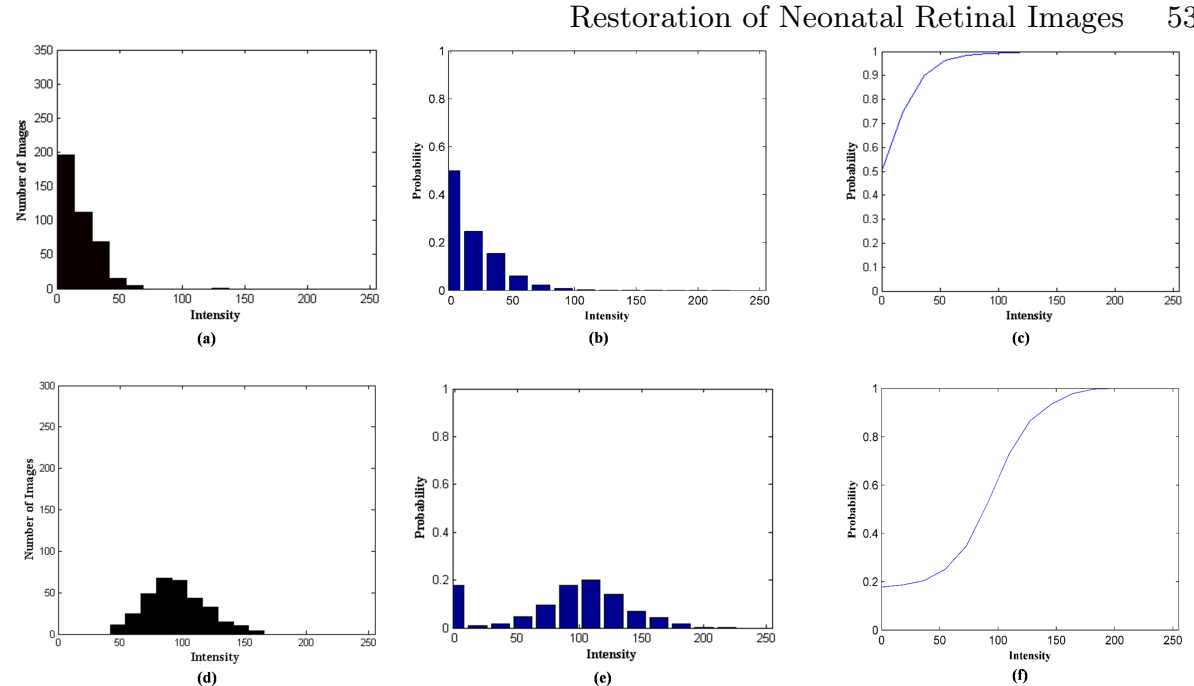

Fig. 3: First and second rows correspond to adult (total 397 images) and neonatal (total 325 images) retinal images respectively. (a) and (d) show average intensity of dark channels of all images, (b) and (e) show histograms of all pixels of dark channels of all images, (c) and (f) show corresponding cdf. The shifts in the dark channels in the second row can be attributed to scattered ambient light because of turbid medium

the restoration problem as imaging through a turbid media. Thus in our model the initial transmission estimate is calculated as

$$
t \tilde{\mathbf{x}})=1-\min _{c \in(r, b)}\left(\min _{\Omega(x)}\left(\frac{I(\mathbf{x})^{c}}{A^{c}}\right)\right)
$$

This is a coarse estimate and hence needs to be refined. Instead of matting which is of high computational complexity, we use guided filter [9] which is an edgeaware operator to refine the transmission map $t(\mathbf{x})$. With coarse transmission map as input and another image as guide, local linear model of guided filter assumes that the filter output to be a linear transform of guidance image in a local window and minimizes a cost function so as to capture the smaller details from guide image while globally maintaining the impression of the input (coarse transmission map). We intend to give an image which provides the knowledge of vasculature as a guide image. The guide image can be given in two waysi)contrast enhanced version of green channel which contains most vasculature information or ii)background extracted statistically from Mahalanobis distance criteria [10]. Mathematically, a given pixel can be said to be in background if the distance

$$
d=\left|\frac{I(\mathbf{x})-\hat{\mu}_{N}}{\hat{\sigma}_{N}}\right|
$$

is lower than a threshold. Here $\hat{\mu}_{N}$ and $\hat{\sigma}_{N}$ are sample mean and sample variance of the input image $I(\mathbf{x})$ respectively. Using this criteria we can separate out the background which contains high vascular information. Example images are shown in Fig. 3. The ambient light is estimated as described previously. Finally, the restored image is given by

$$
J(\mathbf{x})^{c}=\frac{I(\mathbf{x})^{c}+A^{c} t(\mathbf{x})-A^{c}}{t(\mathbf{x}) A^{c}}
$$



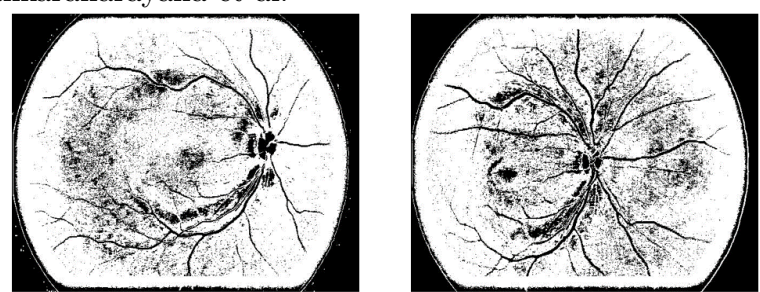

Fig. 4: Example guide images generated from method [10]
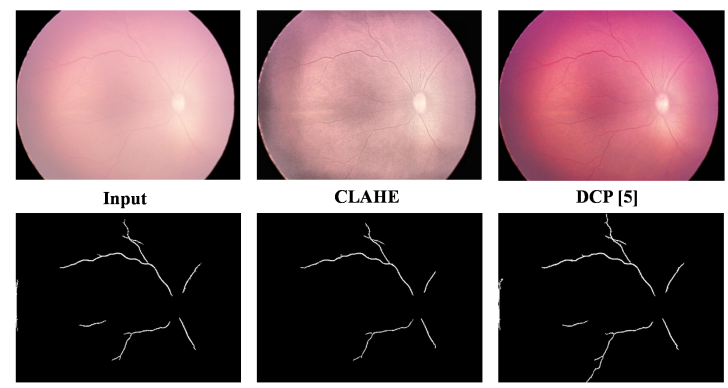

DCP [5]

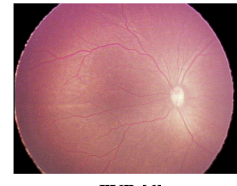

FVR [6]
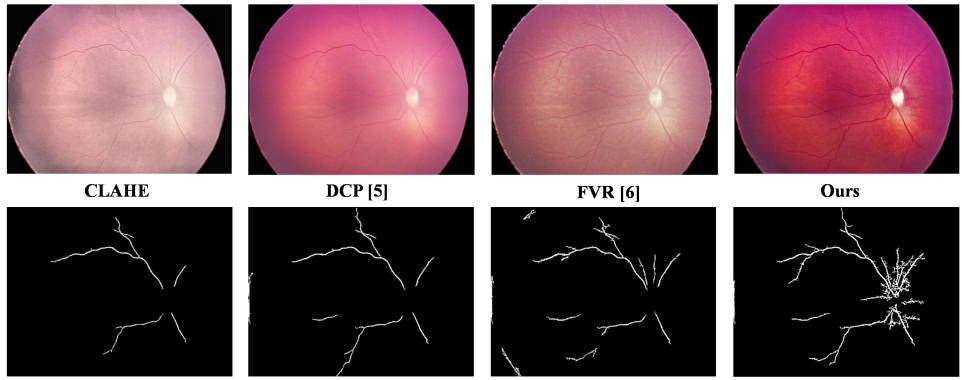

Ours
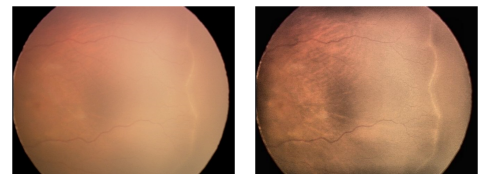

CLAHE

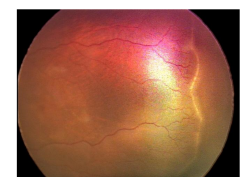

DCP [5]

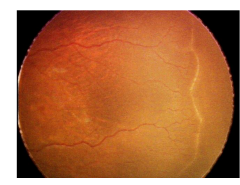

FVR [6]

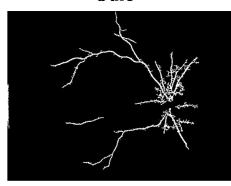

Input
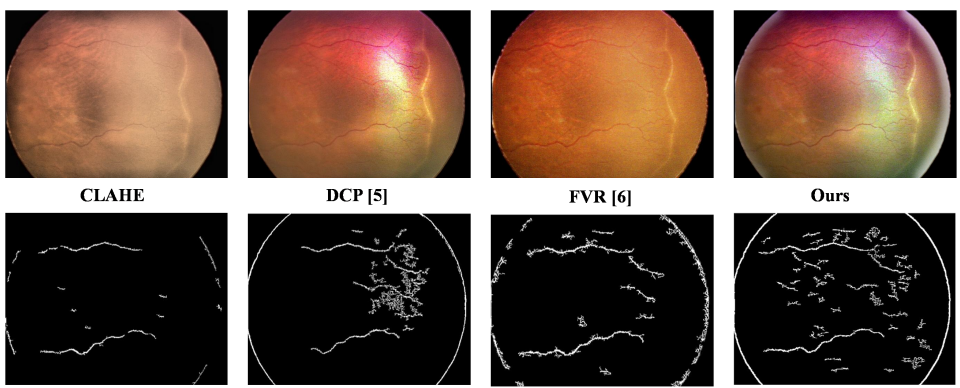

Ours

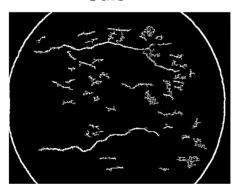

Fig. 5: Dehazing results of various methods for Central View, Stage 1 ROP in the first row and Temporal View, Stage 2 ROP in the third row. Second and fourth rows show the output of vessel segmentation method [2] for different methods.

\section{Experiments}

The effectiveness of the proposed approach is tested using images obtained from a clinical setting. Images were obtained from multiple patients and the ROP disease was categorized to one of the four stages. For each stage, we selected one central and one temporal view of the retinal images randomly from different patients and experimented on different methods. The temporal viewed images were so chosen such that the optic disk was completely absent. We tested our methods along with

1. CLAHE: Contrast limited Adaptive Histogram Equalization applied on the $\mathrm{V}$ channel of HSV after performing the conversion from RGB to HSV.

2. DCP: Dark Channel Prior based dehazing method of He et. al [5]

3. FVR: Fast Visibility Restoration method of Hauterie et. al in [6] 
Table 1: Rate $\boldsymbol{e}$ for Central Views (C $\mathrm{V})$ and Temporal Views (T V) Neonatal Retinal Images of different stages

\begin{tabular}{ccccc}
\hline \hline Stage CLAHE & DCP & FVR & Ours \\
\hline C V & & & & \\
1 & 0.9044 & 0.9422 & 0.9718 & $\mathbf{0 . 9 7 3 7}$ \\
2 & 0.9107 & 0.9308 & $\mathbf{0 . 9 4 1 9}$ & 0.9380 \\
3 & 0.8330 & 0.8587 & $\mathbf{0 . 8 8 5 1}$ & 0.8849 \\
4 & 0.8048 & 0.8331 & 0.9050 & $\mathbf{0 . 9 2 8 0}$ \\
T V & & & & \\
1 & 0.9566 & $\mathbf{0 . 9 7 9 3}$ & 0.9735 & 0.9767 \\
2 & 0.8976 & 0.9225 & 0.9225 & $\mathbf{0 . 9 2 9 1}$ \\
3 & 0.8018 & 0.8057 & 0.8318 & $\mathbf{0 . 8 4 8 8}$ \\
4 & 0.9097 & 0.8927 & 0.9132 & $\mathbf{0 . 9 2 2 0}$ \\
\hline
\end{tabular}

Table 2: Ratio $\boldsymbol{r}$ for Central Views (C $\mathrm{V})$ and Temporal Views (T V) Neonatal Retinal Images of different stages

\begin{tabular}{ccccc}
\hline \multicolumn{2}{c}{ Stage CLAHE } & DCP & FVR & Ours \\
\hline C V & & & & \\
1 & 2.1082 & 2.3695 & 2.9664 & $\mathbf{3 . 1 8 4 2}$ \\
2 & 2.6038 & 2.7029 & $\mathbf{2 . 8 5 8 6}$ & 2.7952 \\
3 & 1.4956 & 1.5035 & 1.6276 & $\mathbf{1 . 6 3 5 3}$ \\
4 & 1.6193 & 1.8176 & 2.1856 & $\mathbf{2 . 3 8 8 1}$ \\
T V & & & & \\
1 & 2.0257 & $\mathbf{2 . 7 7 7 1}$ & 2.4031 & 2.7363 \\
2 & 1.9566 & 2.2317 & 2.1489 & $\mathbf{2 . 2 6 2 8}$ \\
3 & 1.7836 & 1.9869 & 1.9401 & $\mathbf{2 . 1 6 8 8}$ \\
4 & 2.0180 & 1.9386 & 2.2196 & $\mathbf{2 . 3 3 9 4}$ \\
\hline
\end{tabular}

To analyze the outputs of different methods qualitatively, we display the results of two particularly challenging neonatal retinal images among the ones used in our experiment. The first row of Fig. 5 shows the central view of stage 1 ROP image and third row of Fig. 5 shows the temporal view of stage 2 ROP image. It can be seen that the input images are very hazy which makes it difficult for humans and computer systems to do the necessary analysis. CLAHE improves the visibility slightly but much improved results can be obtained from the dehazing techniques with the proposed method offering the most visibility. But in stage 2 ROP case, DCP [5] and our method gives coloring artifact and Visibility Restoration [6] method maintains the color of the input image and is thus more visually pleasing because of its additional step of local white balance processing. The second rows of both the figures show the output of vessel segmentation algorithm [2] for the corresponding methods. Again it can be seen that dehazing based methods perform better than CLAHE and makes vessel segmentation using conventional methods perform much better.

For quantitative analysis, we use two of the metrics used in [6]. We compare the input image and restored image after converting them into gray level. We find the i) the rate $\boldsymbol{e}$ of newly visible edges after restoration calculated based edges in the input image and restored image and ii) the mean ratio $r$ of the gradient norms of edges before and after restoration. Table. 1 shows the values of rate $\boldsymbol{e}$ obtained for various restoration methods. Table. 2 shows the values of mean $r$ and seems to be a better metric for comparison since it gives average increase of contrast on the visible edges. it can be seen that predominantly our method gives the highest values of mean $\boldsymbol{r}$. Based on the table, we can order ours, FVR [6], DCP [5] and CLAHE in the decreasing order of contrast enhancement of edges. This makes our method respond well to vessel segmentation algorithms.

The dehazing method of restoration has an added advantage that apart from enhancement of the image, it also gives depth map, which can be calculated with the help of equation (2). This depth map proves to be very useful in the higher stages of ROP and also to monitor after treatment since it helps in visualizing the retinal surface in $3 \mathrm{D}$ as shown in Fig. 6 

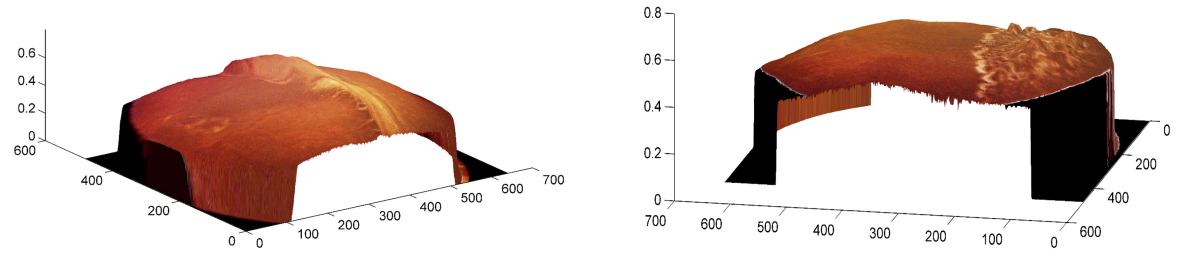

Fig. 6: Retinal 3D surface reconstruction from depth map for (left) ROP in stage 4 and (right) ROP treated in stage 3.

\section{Conclusion}

In this paper, we propose a novel method for the restoration of neonatal retinal images. We compare effectiveness of dehazing techniques in the image enhancement. The results show that dehazing methods perform much better than conventional technique and our method performs better on an average. Moreover, from the proposed method, we also get depth information which can be used to study the retinal structure in 3D. In future, we wish to improve our method by reducing color artifacts and by taking into account spatially varying ambient light intensity.

\section{References}

1. Capone Jr., A. The photographic screening for retinopathy of prematurity study (photo-ROP). Primary outcomes. Retina Today, 2008.

2. Heneghan, C., Flynn, J., O'Keefe, M., and Cahill, M. (2002). Characterization of changes in blood vessel width and tortuosity in retinopathy of prematurity using image analysis. Medical Image Analysis, 6(4), 407-429.

3. Dumont, E.; Hautiere, N.; Gallen, R. A Semi-Analytic Model of Fog Effects on Vision. In Atmospheric Turbulence, Meteorological Modeling and Aerodynamics; Lang, P.R., Lombargo, F.S., Eds.; Nova Science Publishers: New York, NY, USA, 2011; pp. 635-670.

4. Fattal, R. Single Image Dehazing. In ACM SIGGRAPH'08, pages 1-9, New York, NY, USA, 2008. ACM.

5. He, K.; Sun, J.; and Tang, X. Single image haze removal using dark channel prior. IEEE TPAMI, 33(12):2341-2353, 2011.

6. Tarel, J.-P.; Hauti'ere, N. Fast Visibility Restoration from a Single Color or Gray Level Image. In Proceedings of IEEE 12th International Conference on Computer Vision (ICCV 09), Kyoto, Japan, 29 September-2 October 2009; pp. 2201-2208.

7. Carlevaris-Bianco, N.; Mohan, A. and Eustice, R. Initial results in underwater single image dehazing. IEEE OCEANS, pages 1-8, 2010.

8. Drews, P.; Nascimento, E.; Moraes, F.; Botelho, S. and M. Campos, M. Transmission Estimation in Underwater Single Images. Proc. IEEE Int. Conf. Comput. Vis. Workshops (ICCVW), pp. 825-830, Dec. 2013.

9. He, K.; Sun, J.; and Tang, X. Guided image filtering. Proc. Computer Vision ECCV 2010, vol. 6311, pp. 1-14, 2010.

10. Foracchia, M.; Grisan, E. and Ruggeri, A. Luminosity and contrast normalization in retinal images. Med. Image Anal., vol. 9, no. 3, pp. 179-190, 2005 